\title{
Hydrophobic adsorption followed by desorption with ethanol-water for recovery of Penicillin G from fermentation broth.
}

André N. C. de Barros $^{\dagger}$, Emanoela F. Q. Santos ${ }^{\dagger}$, Dasciana S. Rodrigues ${ }^{\dagger, \mathcal{E}}$, Raquel L.

C. Giordano ${ }^{\dagger, \dagger, \alpha}{ }^{*}$, Thiago F. de Pádua ${ }^{\dagger, \alpha}$ *.

$\uparrow$ Graduate Program in Chemical Engineering, Federal University of São Carlos, PO

Box 676, São Carlos, São Paulo, Brazil

$\dagger \dagger$ Chemical Engineering Department, Federal University of São Carlos, PO Box 676,

São Carlos, São Paulo, Brazil

$\alpha$ Both authors contributed equally to this work.

* Correspondence to: $\quad$ Thiago F Pádua (E-mail: tfpadua@gmail.com)

Raquel L. C. Giordano (E-mail: raquel@ufscar.br) 


\section{Supporting information}

\section{Isotherms: parameters for the Freundlich and linear model (from fermentation broth)}

Table S1. Model parameters and correlation coefficient for different adsorption isotherm models (Freundlich and linear) fitted with equilibrium data of PenG/XAD-4 adsorption.

\begin{tabular}{|cc|ccc|cc|}
\cline { 3 - 7 } \multicolumn{1}{c|}{} & \multicolumn{3}{c|}{ Freundlich } & \multicolumn{2}{c|}{ Linear } \\
\hline $\mathbf{p H}$ & $\mathbf{T}\left({ }^{\circ} \mathbf{C}\right)$ & $\mathbf{n}$ & $\mathbf{K}_{\mathbf{F}}$ & $\mathbf{R}^{\mathbf{2}}$ & $\begin{array}{c}\mathbf{K}_{\mathbf{P}} \\
(\mathbf{m L} / \mathbf{m g})\end{array}$ & $\mathbf{R}^{\mathbf{2}}$ \\
& & \multicolumn{3}{|c|}{$\mathbf{q}^{*}=\mathbf{K}_{\mathbf{F}}\left(\mathbf{C}^{*}\right)^{\mathbf{n}}$} & \multicolumn{2}{|c|}{$\mathbf{q}^{*}=\mathbf{K}_{\mathbf{P}} \mathbf{C}^{*}$} \\
& & & & & & \\
\hline $\mathbf{4}$ & $\mathbf{4}$ & $0.615 \pm 0.075$ & $43.42 \pm 10.15$ & 0.968 & $10.81 \pm 1.41$ & 0.828 \\
$\mathbf{7}$ & $\mathbf{4}$ & $0.593 \pm 0.089$ & $44.79 \pm 12.42$ & 0.950 & $10.14 \pm 1.51$ & 0.891 \\
$\mathbf{4}$ & $\mathbf{1 2}$ & $0.401 \pm 0.067$ & $97.39 \pm 19.29$ & 0.935 & $10.48 \pm 2.45$ & 0.785 \\
$\mathbf{7}$ & $\mathbf{1 2}$ & $0.458 \pm 0.046$ & $71.41 \pm 10.13$ & 0.980 & $9.45 \pm 1.65$ & 0.830 \\
\hline
\end{tabular}




\section{Isotherms: Sum of square and mean square}

Table S2. Model parameters and correlation coefficient for different adsorption isotherm models (Freundlich and linear) fitted with equilibrium data of PenG/XAD-4 adsorption.

\begin{tabular}{|c|c|c|c|c|c|c|c|c|c|c|c|c|c|}
\hline & & \multicolumn{4}{|c|}{ Langmuir model } & \multicolumn{4}{|c|}{ Freundlich } & \multicolumn{4}{|c|}{ Linear } \\
\hline & $\begin{array}{l}\text { Source of } \\
\text { variation }\end{array}$ & $\begin{array}{l}\text { Sum of } \\
\text { squares }\end{array}$ & $\begin{array}{l}\text { Degrees of } \\
\text { freedom }\end{array}$ & $\begin{array}{l}\text { Mean } \\
\text { Square }\end{array}$ & $\mathrm{F}_{\text {calc }} *$ & $\begin{array}{l}\text { Sum of } \\
\text { squares }\end{array}$ & $\begin{array}{l}\text { Degrees of } \\
\text { freedom }\end{array}$ & $\begin{array}{l}\text { Mean } \\
\text { Square }\end{array}$ & $\mathrm{F}_{\text {calc }}{ }^{*}$ & $\begin{array}{l}\text { Sum of } \\
\text { squares }\end{array}$ & $\begin{array}{l}\text { Degrees of } \\
\text { freedom }\end{array}$ & $\begin{array}{l}\text { Mean } \\
\text { Square }\end{array}$ & $\mathrm{F}_{\text {calc }} *$ \\
\hline & Regression & 112800 & 1 & 112800 & 879.0 & 109813 & 1 & 109813 & 236.5 & 103743 & 1 & 103743 & 24.1 \\
\hline & Residual & 641.6 & 5 & 128,3 & & 2321.4 & 5 & 464.3 & & 21506 & 5 & 4301.3 & \\
\hline & Regression & 103226 & 1 & 103226 & 431.4 & 101583 & 1 & 101583 & 152.8 & 133903 & 1 & 133903 & 32.6 \\
\hline & Residual & 1196.3 & 5 & 239.3 & & 3324 & 5 & 664.8 & & 16452.4 & 5 & 4113.1 & \\
\hline \multirow{2}{*}{\begin{tabular}{ll} 
子 & \multicolumn{1}{c}{} \\
$\frac{1}{2}$ & $\stackrel{2}{2}$
\end{tabular}} & Regression & 120143 & 1 & 120143 & 1161.3 & 112678 & 1 & 112678 & 124.9 & 198243 & 1 & 198243 & 18.3 \\
\hline & Residual & 517.3 & 5 & 103.5 & & 4509 & 5 & 901.8 & & 54263.7 & 5 & 10852.7 & \\
\hline \multirow{2}{*}{ 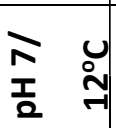 } & Regression & 105117 & 1 & 105117 & 2749.6 & 100394 & 1 & 100394 & 336.0 & 149930 & 1 & 149930 & 20.2 \\
\hline & Residual & 191.1 & 5 & 38.3 & & 1494.0 & 5 & 298.8 & & 29708.7 & 5 & 7427.2 & \\
\hline
\end{tabular}

${ }^{*} F_{\text {tab,regress }}=6.61$ 


\section{First experimental design: ANOVA and surface response}

Table S3. - ANOVA for second-degree polynomial model in the first experimental design.

\begin{tabular}{lccccc}
\hline SOURCE & $\begin{array}{c}\text { Sum of } \\
\text { squares }\end{array}$ & $\begin{array}{c}\text { Degrees of } \\
\text { freedom }\end{array}$ & $\begin{array}{c}\text { Mean } \\
\text { square }\end{array}$ & F & p-value \\
\hline T(linear) & 3.77 & 1 & 3.77 & 2 & 0.2524 \\
T(quadratic) & 194.22 & 1 & 194.22 & 102.9 & 0.002 \\
pH(linear) & 28.01 & 1 & 28.01 & 14.84 & 0.0309 \\
pH(quadratic) & 312.93 & 1 & 312.93 & 165.79 & $<0.001$ \\
[EtOH](Linear) & 3873 & 1 & 3873 & 2051.88 & $<0.001$ \\
[EtOH]( quadr) & 842.85 & 1 & 842.85 & 446.53 & $<0.001$ \\
T x pH & 2.02 & 1 & 2.02 & 1.07 & 0.377 \\
T x [EtOH] & 1.04 & 1 & 1.04 & 0.55 & 0.5124 \\
pH x [EtOH] & 62.94 & 1 & 62.94 & 33.35 & 0.0103 \\
RESIDUAL & 535.85 & 8 & 66.98 & & \\
\hline TOTAL & $\mathbf{5 5 2 7 . 2 9}$ & $\mathbf{1 7}$ & & & \\
\hline \multicolumn{1}{c}{$\mathbf{R}^{\mathbf{2}}$} & \multicolumn{7}{c}{$\mathbf{F}_{\text {(regress/res)Calc }}$} \\
\multicolumn{7}{c}{ 90.31\% } & & 8.28 & & F \\
\hline
\end{tabular}
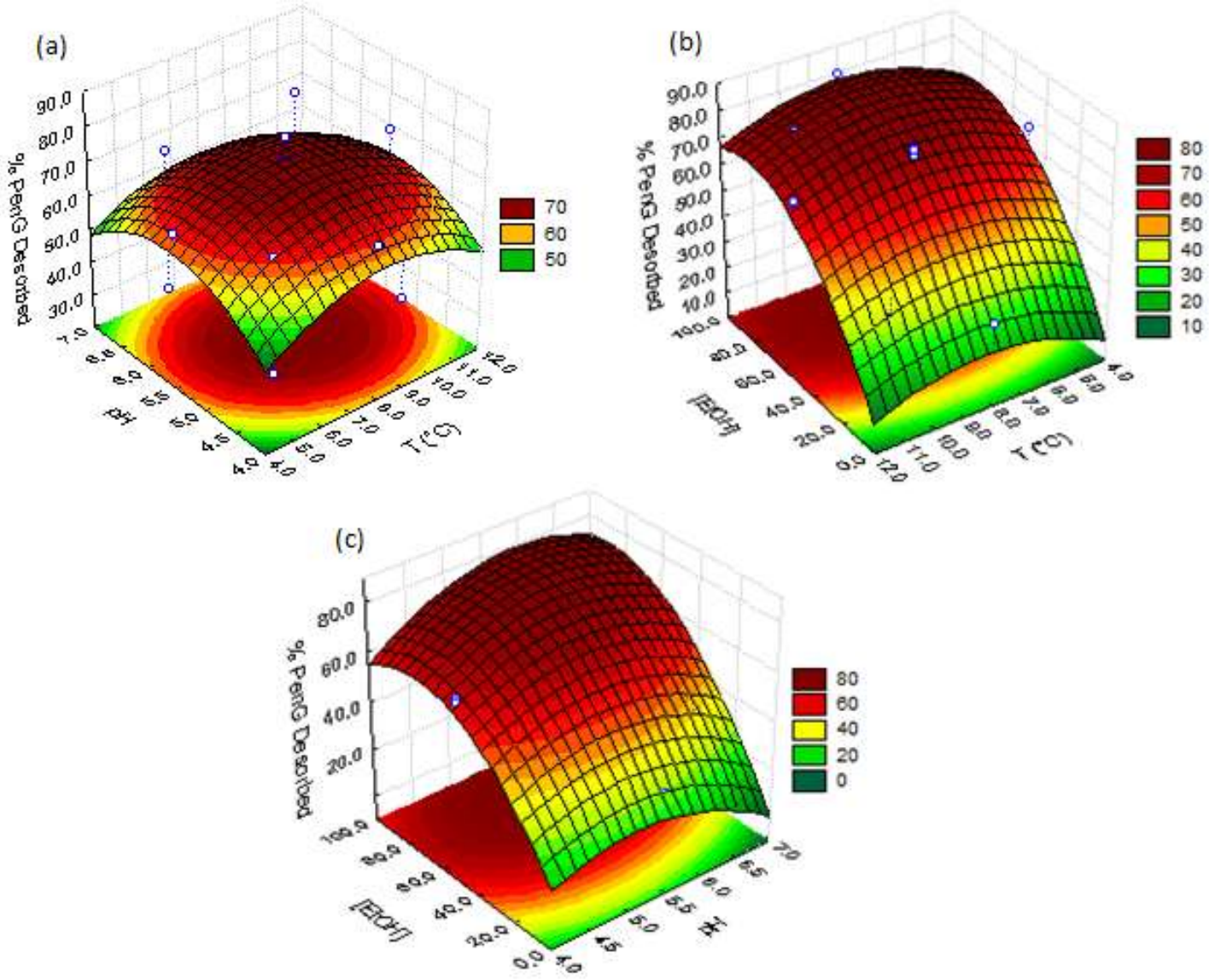

Figure S1. Second-degree polynomial model for the percentage of desorbed PenG of the first design as a response of (a) Temperature and $\mathrm{pH}$; (b) Temperature and ethanol concentration (\% of mass). (c) $\mathrm{pH}$ and ethanol. 
4. Second experimental design: ANOVA and surface response

Table S4. - ANOVA for the second polynomial model evaluated (second design).

\begin{tabular}{|c|c|c|c|c|c|}
\hline SOURCE & $\begin{array}{l}\text { Sum of } \\
\text { squares }\end{array}$ & $\begin{array}{c}\text { Degrees of } \\
\text { freedom }\end{array}$ & $\begin{array}{l}\text { Mean } \\
\text { square }\end{array}$ & $\mathbf{F}$ & p-value \\
\hline pH(Linear) & 4.969 & 1 & 4.968 & 0.137 & 0.720 \\
\hline pH(quadratic) & 221.03 & 1 & 221.029 & 6.122 & 0.038 \\
\hline [EtOH](Linear) & 7.282 & 1 & 7.282 & 0.201 & 0.665 \\
\hline [EtOH]( quadr) & 252.347 & 1 & 252.347 & 6.989 & 0.029 \\
\hline pH x [EtOH] & 3.940 & 1 & 3.940 & 0.109 & 0.749 \\
\hline RESIDUAL & 288.813 & 8 & 36.101 & & \\
\hline TOTAL & $\begin{array}{c}1115.98 \\
4\end{array}$ & 13 & & & \\
\hline $\begin{array}{c}\mathbf{R}^{\mathbf{2}} \\
74.12 \% \\
\end{array}$ & & & $\begin{array}{c}\mathbf{F}_{\text {(regress/res) }} \\
\text { Calc } \\
4.58 \\
\end{array}$ & & $\begin{array}{c}\mathbf{F}_{\text {tab }} 95 \% \\
3.69 \\
\end{array}$ \\
\hline
\end{tabular}

